\title{
Jenseits des „Blue Hole": Zur Konsolidierung der Meere in der Geschichtswissenschaft
}

Franziska Torma

Mareike Vennen 2018. Das Aquarium. Praktiken, Techniken und Medien der Wissensproduktion (1840-1910). Göttingen: Wallstein Verlag, geb. 423 S., € 37,00, ISBN: 978-3-83533-252-2.

Carmel Finley 2017. All the Boats on the Ocean. How Government Subsidies Led to Global Overfishing. Chicago: Chicago University Press, geb. 224 S., US\$ 45,00, ISBN: 978-0-22644-337-9.

Jens Ruppenthal 2018. Raubbau und Meerestechnik. Die Rede von der Unerschöpflichkeit der Meere. Stuttgart: Franz Steiner Verlag, geb. 293 S., € 56,00, ISBN: 978-3-515-12121-7.

Helen M. Rozwadowski 2018. Vast Expanses A History of the Oceans. London: Reaktion Book, geb. 264 S., f 16,00, ISBN: 978-178023-997-2.

Vor knapp zehn Jahren hätte ein Rezensionsessay zur Rolle der Meere und Ozeane ${ }^{1}$ in den Geschichtswissenschaften noch mit der Beschwörung des Blue Hole begonnen. Der amerikanische Historiker John Gillis brachte die marine Forschungslücke für die Umweltgeschichte prägnant auf den Punkt: „There is a blue hole in environmental history. It remains a remarkably landlocked discipline, one that largely ignores the seven-tenths of our globe's surface covered by water and an ecosystem estimated to constitute ninety-eight percent of our biosphere" (Gillis 2011: 16). Im Jahr 2019 heißt es dagegen: „Die Geschichte des Meeres ist in letzter Zeit populär geworden" (Ruppenthal et al. 2019: 9). 
Angeregt von Pionierarbeiten aus dem angloamerikanischen Raum (Bolster 2006; Bolster 2012; Chiarappa \& McKenzie 2013), erleben die Ozeane nun auch in der deutschsprachigen Historiographie einen Bedeutungsgewinn: Dies betrifft sowohl Monographien, (etwa Sparenberg 2012) als auch Themenhefte und Sammelwerke (etwa Kraus \& Winkler 2014a). Das Spektrum reicht Von Flaschenpost bis Fischreklame, wie der Titel eines lesenswerten Bandes verheißt, der auf einem Workshop zur Wahrnehmung des Meeres entstanden ist (Ruppenthal et al. 2019). Ökologische Gründe tragen zum Bedeutungsgewinn bei: Artensterben, Meeresverschmutzung, Klimawandel und der weltweite Anstieg des Meeresspiegels erzeugen eine umweltpolitische und umwelthistorische Brisanz.

Ozeane ermöglichen auch methodische Innovation und historiographische Neuakzentuierung. In der Ozeanographie ist das Blue Hole ein Senkloch in einem Riff. Diese doppelte Verwendung zeigt, dass das Meer Geschichtsraum und zugleich materielle Umwelt ist (Steinberg 2001). In Form der Tiefe wird die dritte Dimension des Planeten zum Forschungsgegenstand, wobei der schwer zugängliche Raum unter der Wasseroberfläche die Ambivalenzen der Moderne (Bauman 2016) verdichtet. Anhand der Ozeane kann neu überlegt werden, welche Prozesse und Faktoren die terrestrische Moderne charakterisieren, und wie sich andere Umwelten vom gewohnten Terrain unterscheiden. Die Untiefen des Blue Hole bündeln die Faszination, aber auch die Unsicherheit, was in den blauen Zonen und an den Rändern der menschlichen Geschichte geschehen ist. Im Meer spiegeln sich Nicht-Wissen, Spekulationen und Vermutungen in planetarischem Maßstab; es vereint Wissenschaft mit Möglichkeiten des Denkens. Mark Chiappara und Matthew McKenzie vertraten vor einigen Jahren die These, dass die moderne Wissenschaft, mit ihrem Hang zur Technisierung und Objektivierung, in der fluiden, maritimen Umwelt an ihre epistemischen und praktischen Grenzen geriet (Chiarappa \& McKenzie 2013: 9). ${ }^{2}$ Für ihr Spezialgebiet der marine environmental history zeigen sie, wie sich menschliche Eingriffe in die Meeresumwelt durch vermeintliche Erfolgsansprüche legitimieren ließen. An der schwer einzuhegenden und leicht zu zerstörenden Natur des Meeres scheiterten jedoch die Heilsversprechen der Moderne.

Vor dem Hintergrund oben skizzierter Debatten, beginnt sich das Meer in den letzten Jahren als historischer Forschungsbereich zu konsolidieren. ${ }^{3}$ Die Beschwörung diverser turns wie dem maritime turn, dem oceanic turn (siehe Kraus \& Winkler 2014b), die Suche nach Konzepten wie seascapes (siehe Wigen 2007: 1-18) und die Entwürfe von Forschungsagenden werden zunehmend von empirischen Studien abgelöst. Nicht mehr alleine die Frage, was mit welchen Begriffen und Methoden getan werden könnte und sollte, okkupiert den wissenschaftlichen Buch- und Zeitschriftenmarkt. ${ }^{4}$ 
Neu erscheinende Studien geben den Meeren und Ozeanen scharfe, historische Konturen. Die vier besprochenen Bücher verbinden wissenschaftsund technikhistorische mit kulturhistorischen, politikhistorischen und umwelthistorischen Perspektiven.

\section{Wissensfigur "Aquarium": Die Schnittmenge von science and technology studies (StS) und Kulturwissenschaft}

Mareike Vennens Buch Das Aquarium. Praktiken, Techniken und Medien der Wissensproduktion (1840-1910) zeichnet sich positiv durch methodologische Experimentierfreude und Vielschichtigkeit aus. Die luzide Argumentation und der klare Schreibstil geleiten die Leser*innen sicher (und mit Vergnügen) durch die Materie. Vennens Buch liefert einen originellen Beitrag zur seit Jahren laufenden Aquarienforschung. ${ }^{5}$ Diese Studie steht an der Schnittstelle von Science and Technology Studies und Kulturwissenschaft. Zentral ist das Motiv des Wissens und der Wissensfiguren (ähnlich auch am Beispiel des Wals, Lüttge 2016). Im Sinn des interdisziplinären Denkhorizonts neuer objektbasierter Forschung steht nicht eine spezifische analytische Methode, sondern das Aquarium als Ding im Zentrum. Um dieses von Tieren bewohnte Glasgebilde „verdichten sich die naturkundlichen Wissenswelten des 19. Jahrhunderts“ (S. 10). Anhand der frühen Aquariengeschichte entwickelt die Autorin fünf weiterführende Arbeitshypothesen:

1. Wissenshistorisch betrachtet, war das Aquarium nicht nur „ein dekoratives Zierobjekt im bürgerlichen Salon“, sondern „ein epistemisches Objekt ersten Ranges." (S. 9-10).

2. Für die sich etablierende Aquaristik war die Benutzungsgeschichte (dirty hard work) zentral. Wie bei der Tierhaltung im Zoologischen Garten, töteten Aquarienbesitzer die Tiere selten zu Forschungs- oder Prestigezwecken. Der Sinn des Aquariums bestand gerade darin, ihnen ein Milieu zum (Über-)Leben zu bauen. Die Aquarien waren somit „Wissensumwelten im Glas" (S. 10), die durch menschliche Arbeit gestaltet waren.

3. Aquarien waren aber auch „mediale Dispositive der Wissensproduktion, die das Wissen und die Vorstellungen über das Leben unter Wasser nachhaltig veränderten" (S. 11). Sie beeinflussten damit die naturkundliche Forschung, wie, wo und womit diese durchgeführt wurde. Sie prägten auch populäre Vorstellung vom Meer und dessen Leben.

4. Aquarien waren spezifische Orte innerhalb der Geografie der Wissenschaften. Sie entstanden in Zwischen-, Verkehrs- und Transiträumen jen- 
seits der etablierten Institutionen. Diese Zwischenlage sensibilisiert für die infrastrukturellen Voraussetzungen der Aquaristik, zum Beispiel für Verpackungs- und Mobilisierungspraktiken.

5. Weder Verwendungszwecke, noch Materialität waren in der frühen Aquaristik eindeutig festgeschrieben. Auch die Trennung zwischen Liebhaberei und Forschung war nicht klar markiert. Anhand des Aquariums wird deshalb deutlich, wie naturkundliches, chemisches und ingenieurstechnisches Wissen jenseits der universitären Ordnung der Disziplinen zusammenhängt. Dieses Mischverhältnis zeigt eindringlich, dass Interdisziplinarität bereits um 1900 als Praxis erforderlich war. Um dem Aquarium als Gegenstand gerecht zu werden, verknüpft Vennen wissensgeschichtliche, medien- und kulturwissenschaftliche Ansätze.

Der Fluchtpunkt der Untersuchung liegt in der Entstehung ökologischen Wissens. Damit fügt Vennen überzeugend Lynn Nyharts biologiehistorischer These vom Wandel der Naturgeschichte zur Lebenswissenschaft im 19. Jahrhundert (Nyhart 2009) eigene, innovative Facetten hinzu: Sie fokussiert auf die Praktiken, Techniken und Medien, mit denen Amateure im Aquarium ökologisches Wissen avant la lettre erzeugten. Die Hobbynaturalisten (und die sich parallel professionalisierende Aquarienindustrie) lernten an ihrem Liebhaberobjekt, wie Beziehungen und Wechselwirkungen zwischen den Lebewesen (auch: Mensch/Tier) und der Natur idealerweise auszusehen haben. Am prekären Zustand des ökologischen Gleichgewichts entfalteten sich mitunter Prozesse von Vereinnahmung und Widerständigkeit. Daraus entstanden die Wissensfiguren des „balanced aquariums" und des "circulated aquariums" (S. 63-81 bzw. 215-234). Einerseits spiegelt sich darin eine Erfolgsgeschichte technischen Fortschritts wider, andererseits eine Krisengeschichte, die offenlegte, was alles schiefgehen konnte.

Das Aquarium zeigt die menschliche Faszination am Meer. Untersuchungen zur Fischerei demonstrieren dagegen das menschliche Zerstörungspotential. Hier ließen die Lerneffekte Jahrzehnte auf sich warten. Bewunderung und Zerstörung machten grundsätzliche Spannungsfelder der menschlichen Beziehung zum Meer aus.

\section{Trawlerflotten zwischen internationalen Beziehungen und Fischereigeschichte}

Warum nahm die Fischerei im Verlauf des 20. Jahrhunderts derartig desaströse Ausmaße an? Carmel Finleys äußerst dichte, gut lesbare Studie 
zur Expansion der Weltfischerei nach dem Zweiten Weltkrieg widmet sich dem Problem der Überfischung und der damit verbundenen Vernichtung aquatischer Ökologien. Mit der Fischereigeschichte vermisst sie einen etablierten marinen Forschungsbereich neu (etwa Heidbrink 2004; Hubbard 2006). An den prägnanten Titel des Buches, „All the Boats on the Ocean“, schließt sie in der Einleitung die Frage an: „who built all the boats in the first place?" (S. 2).

Der Prototyp dieser neuen Generation von industriellen Fangschiffen stand auf einer schottischen Werft in Aberdeen. Dort glitt im Jahr 1954 der 280 Fuß lange Trawler Fairtry ins Wasser. Mit einem herkömmlichen Fischerboot hatte er wenig gemein. Er sah aus wie eine Mischung aus Kreuzfahrt- und Walfangschiff. Die Fairtry revolutionierte den internationalen Fischfang. Sie verband moderne Fischfangtechnologie, Fischverarbeitung und Tiefkühlung. Sie konnte wochenlang auf See bleiben und sogar unter stürmischen Bedingungen mit hoher Geschwindigkeit fischen. In nur 37 Tagen verarbeitete die Crew 2000 metrische Tonnen Kabeljau zu 650 metrischen Tonnen Kabeljaufilet.

Das Schiff selbst war einerseits das Resultat von sechsjährigen Experimenten der britisch-norwegischen Walfanggesellschaft Christian Salvesen. Andererseits wies es als Blaupause des Trawlerbaus in die Zukunft. Ziemlich bald bestellte die Sowjetunion 20 dieser Schiffe. Das setzte eine weitere Innovationswelle in Gang: „Modern technology had been introduced in fisheries elsewhere, ,but the translation of the ideas into large-scale practice is a wholly Soviet achievement."“ (S. 93) Die hochtechnisierten sowjetischen Fischfangflotten waren gigantisch in der Größe, sie fischten global und zeigten dabei, wieviel an Ausbeute mit perfektionierter Technologie möglich war. Dabei blieben sie vom gleichzeitig entstehenden Konzept des maximum sustainable yield, dessen amerikanische Geschichte die Autorin in ihrem ersten Buch All the Fish in the Sea (Finley 2011) behandelte, ziemlich unbeeindruckt. Laut Finley hielten sich die sowjetischen Flotten nicht an internationale Regeln und Codices. Sie jagten außerhalb der Saison und missachteten Schonfristen für Mütter und Walkälber: „Wastage was systemic, with more whales and fish being killed than could be processed before they spoiled." (S. 94) Finley stellt damit die Frage nach der globalen Reichweite von Konzepten wie dem maximalen nachhaltigen Ertrag, wobei offen bleibt, inwieweit sich die Praktiken der anderen Fischfangnationen vom sowjetischen Vorgehen unterschieden (Holm 2012: 66-91). War es nur eine Abstufung in Ausmaß und Quantität oder doch in der Qualität der Fangpraktiken? Vermutlich traf die erste Variante zu: So übermittelten die Japaner, die zeitgleich zur Entstehung dieses Essays den kommerziellen Walfang wieder aufgenommen haben, wie die Sowjets frisierte Fangzahlen an die International Whaling Commission. 
Finley gibt jedoch eine im internationalen Rahmen gültige Antwort auf die Frage, warum sich kostspielige, mechanisierte Trawlerflotten über die Weltmeere ausbreiteten. Regierungssubventionen waren für den forcierten Schiffbau und die daraus resultierende Überfischung verantwortlich. Damit knüpft Finley Spezialaspekte der Fischereigeschichte an die großen Fragen internationaler Beziehungen (Dorsey 2014). Finley zeigt auf, dass Staatsakte und Schlüsselereignisse wie die Potsdamer Erklärung, die Truman-Doktrin und der Koreakrieg eng mit Fischereifragen verknüpft waren. Der Kalte Krieg verband sich mit den Herausforderungen der Dekolonisation und des heraufziehenden Nord-Südproblems: Die Lösung des Welthungers (Cullather 2010), mitunter durch Fischfang, sollte für soziale Stabilität sorgen, die Entwicklung des Welthandels für Weltfrieden. Trotz dieser humanitären Motive bezeichnet Finley den industrialisierten Fischfang als Form des Imperialismus, der sich nach dem Wegfall überseeischer Kolonien auf die Unterwasserwelt verlagerte.

Verdienstreich ist, dass sich Finley mit dem Pazifik auf einen Schauplatz konzentriert, der in der Fischereigeschichte kaum beleuchtet worden ist. In acht Kapiteln führt das Buch durch zentrale Spannungsfelder der Nachkriegspolitik. Fische tauchen als politische Akteure auf, deren Vorkommen und Verschwinden internationale Vertragswerke strukturieren. Vor allem eine Rotbarschspezies (rosefish), den sowjetische Fischfangflotten vor der Küste Oregons ausgelöscht haben sollen, zeigt verdichtet den hohen Preis industrialisierter Fischerei und die ökologischen Lasten des Kalten Krieges.

Das Buch ist nicht nur durch analytisches Geschick und historiographische Verve geprägt. Auch ein gewisser Aktivismus ist zwischen den Zeilen $\mathrm{zu}$ erkennen. Unterschwellig ist die Aufforderung $\mathrm{zu}$ einem verträglicheren Handeln für die Zukunft herauszulesen. Indem Finley die politischen Fehlentwicklungen der Vergangenheit auffächert, fordert sie auf, aus dieser Geschichte für den zukünftigen Umgang mit den globalen Fischbeständen zu lernen.

\section{Fisch und Mangan: Umweltgeschichte und maritime history}

Jens Ruppenthals Studie Raubbau und Meerestechnik widmet sich ebenfalls der Fischerei, jedoch unter der Perspektive der Ressourcenausbeutung. Er untersucht Fisch und mineralische Rohstoffe. Dabei leistet er einen Beitrag zur Globalgeschichte der Bundesrepublik. Die Studie verbindet Umweltgeschichte und maritime history für den deutschen Fall gekonnt, und bezieht kulturhistorische Perspektiven mit ein. 
Nach einem ausführlichen Forschungsüberblick und Erläuterungen zum rechtlichen Status der See stehen in zwei Großkapiteln die lebenden Ressourcen und die mineralischen Rohstoffe im Zentrum. Das letzte Kapitel fasst die Ergebnisse zusammen und liefert einen Ausblick. Die Entwicklung der bundesrepublikanischen Ressourcendebatte wird von der internationalen Seerechtsfrage gerahmt. Ruppenthal denkt dabei in der longue durée, indem er die ersten Seerechtsdebatten im 16. Jahrhundert reflektiert. Dieser diachrone Bezug verweist darauf, dass der rechtliche Status der Meere seit der Frühen Neuzeit ein Dauer-Streitpunkt war.

Im 20. Jahrhundert gewann das internationale Seerecht auf dem Höhepunkt des Kalten Krieges an Brisanz. Im Jahr 1967, als ein technischer Defekt des amerikanischen Frühwarnsystems fälschlicherweise für einen sowjetischen Angriff gehalten wurde, der die Welt an den nuklearen Abgrund katapultierte, hielt der maltesische Diplomat Arvid Pardo (1914-1999) eine Rede vor der Generalversammlung der Vereinten Nationen. Diese beschwor das Ideal des „Common Heritage of Mankind“ (Hardin 1968; Höhler 2014). ${ }^{6}$ Pardo wandte sich gegen die Verwendung der Meere als Transitraum nuklear aufgerüsteter Flotten, gegen Dumping, Überfischung und den Abbau mineralischer Ressourcen. Die Meere und der Meeresboden jenseits der nationalen Hoheitsgewässer seien Teil des gemeinsamen Erbes der Menschheit, so Pardo. Er trat damit als Fürsprecher der Natur, aber noch vielmehr als Anwalt der sogenannten Entwicklungsländer auf. Eine eigens einzurichtende Behörde sollte die marine Ressourcenausbeutung überwachen, um sicherzustellen, dass nicht nur die Staaten, die über Kapital und Technik verfügten, den Löwenanteil der Meeresschätze an sich nähmen. Ängste vor einer Wiederholung der Ausbeutung des Landes in den Jahren des Kolonialismus und Imperialismus schimmerten durch.

Das Ideal der Gemeingüter hatte große symbolische Ausstrahlung, doch die formale Erklärung zum common heritage of mankind zog sich über Jahrzehnte hin und wurde im Zuge der Seerechtskonferenzen der Vereinten Nationen entwickelt (UNCLOS I-III, 1958-1982; dazu: Sackel 2017; Freestone 2006; Anand 1983). Ruppenthal fokussiert besonders auf den Stellenwert, den die lebenden und mineralischen Ressourcen im Verrechtlichungsprozess für die Bundesrepublik einnahmen. Die Bundesrepublik verfügte nämlich nur über eine kurze Küstenlinie an der Nord- und Ostsee, was die Zugriffsmöglichkeiten auf die Fische und mineralischen Rohstoffe auf den räumlich marginalen Kontinentalsockel reduzierte. Damit berührt Ruppenthal ein Kerndilemma der bundesrepublikanischen Suche nach einem Platz in der marinen Welt: Zusammenarbeit diente dem Zweck, die deutschen Interessen an den internationalen Ansprüchen auf die Fischbestände und den Meeresbergbau zu sichern. 
Der Fall der Fischerei zeigt darüber hinaus historische Widersprüche der aktuellen Nachhaltigkeitsdebatte: Bereits zu Beginn des 20. Jahrhunderts kam die Rede vom sogenannten Raubbau an den Fischbeständen auf. Die Überfischung war in der Fischereiwissenschaft bereits seit Beginn des 20. Jahrhunderts Diskussionsgegenstand. Doch erst in den 1950er und 1960er Jahren säten mediale Kampagnen öffentliche Zweifel an der Unerschöpflichkeit der Meere. Der Fall des Meeresbergbaus zeigt dagegen, wie sich der Kurzküstenstaat Deutschland Anteile an den wertvolleren, mineralischen Ressourcen verschaffen wollte. Mit dem Meeresbergbau war ein immenser technischer Aufwand verbunden, der eine Koalition aus Staat, Industrie und Wissenschaft erforderte. Wissenschaftliche Vereinigungen und Forschungseinrichtungen betonten im Verlauf des 20. Jahrhunderts die Notwendigkeit, das Meer zu nutzen. Der Knackpunkt der Studie liegt nun darin, dass Ruppenthal mit dem Thema der Meeresressourcennutzung einen wenig thematisierten Bereich untersucht. Zwar sind die Holznot und damit verbundene Debatten vergleichsweise gut bekannt, doch die Ressourcennutzung im Meer ist immer noch ein Randthema. Gerade das Tarieren zwischen Ausbeutung und Schonung machte das Gleichgewicht von Ökologie und Ökonomie zum Gegenstand. Beides müsse sich die Waage halten, damit die Fischwirtschaft nachhaltig wirtschaften könne. Trotzdem galten Fische, im Gegensatz zu den Erzen, lange Zeit als nachwachsende Ressourcen, denen Ausbeutung im großen Stil wenig anhaben könne. Die Rede von der Unerschöpflichkeit der Fischbestände ist deshalb als diskursiver Ausblendemechanismus zu verstehen: Die Schäden der Überfischung machten sich zwar bemerkbar; diese als Tatsache anzuerkennen, hätte aber die Veränderung eines gesamten Wirtschafts- und Konsumzweigs bedeutet.

\section{Leisure Frontier und fragile Umwelt: Ein Modell für ocean history}

Bereits seit den 1960er und 1970er Jahren hätten die Zeitgenoss*innen es ahnen können: Der Ozean ist ein bedrohter, schützenswerter Raum. In den 1950er Jahren dehnte sich nicht nur der Fischfang aus; es begannen auch Prozesse der massenmedialen Vermittlung und der freizeittauglichen Aneignung der Unterwasserwelt. Warum dauerte es jedoch gut fünfzig Jahre, bis eine öffentliche, umweltpolitische Sensibilität für die Meere aufkam?

Diese Frage setzt Helen Rozwadowskis Buch Vast Expanses. A History of the Oceans den Leser*innen in den Kopf. Konzipiert mitunter als Überblickswerk, das aufgrund der guten Nachvollziehbarkeit das Potential zu einem textbook für den Unterricht oder gar zum Publikumsbuch hat, er- 
zählt sie die Geschichte des Ozeans. Dieser Kollektivsingular ist vom historiographischen Ziel geleitet, ein Modell für zukünftige Forschungsarbeiten $\mathrm{zu}$ liefern.

Das erste Kapitel beginnt vier Milliarden Jahre vor unserer Zeitrechnung. Rozwadowskis Helden der Urzeit sind nicht die Dinosaurier, sondern die Mollusken. Das zweite Kapitel lotet die frühen menschlichen Beziehungen zum Ozean aus. Manche Kulturen begriffen die See als Teil ihrer Welt, andere wandten sich bewusst von ihr ab. Die Kapitel drei und vier beleuchten das 15.-19. Jahrhundert (dazu auch: Mackay 2018; Rozwadowski 2005). Zunächst stand im Zeitalter der geographischen Entdeckung die horizontale Erschließung der Meere im Zentrum. Diese ozeanischen Routen bildeten die Basis der frühneuzeitlichen Kolonialreiche, die ihre Macht und ihren Reichtum auf die Beherrschung der See gründeten. Im 19. Jahrhundert setzte schließlich die vertikale Erkundung der See ein. Mit der Entdeckung der dritten Dimension verwandelte sich die Welt unter Wasser in einen Forschungs- und Wirtschaftsraum, sowie eine Arena transozeanischer Kommunikation. Damit wurde der Ozean zu einem soziokulturellen Referenzpunkt und Raum menschlicher Zugriffsmöglichkeiten. Eine davon, der ökonomische Wert des Meeres, steht im fünften Kapitel im Vordergrund. Technische Entwicklungen während der Weltkriege brachten die Industrialisierung in das Meer. Dadurch weitete sich die menschliche Beziehung zum Ozean räumlich in der Tiefe aus: Das sechste Kapitel zeigt, wie eine Gruppe von Unternehmern, Erfindern und Offiziellen die Metapher der frontier - eigentlich mit der Erschließung des amerikanischen Westens konnotiert (Turner 1894; Rozwadowski 2012; Cowan 1960) - seit den 1950er Jahren auf die wirtschaftliche Öffnung der See übertrugen. Daran anknüpfend entwickelten sich großangelegte Utopien von Unterwasserhabitaten, in denen Menschen leben und arbeiten konnten. Im siebten Kapitel zeichnet Rozwadoski nach, wie Taucher, Filmemacher und die Ölindustrie die Unterwasserwelt als leisure frontier besetzten und damit eine Verwandlung der robusten Ausbeutungsfrontier zur zerbrechlichen Umwelt einläuteten. Eine bedeutende Rolle spielte die Faszination an der charismatischen Megafauna (etwa Wale) sowie der Schock und die Empörung über Verschmutzungskatastrophen (Ölverschmutzung). Diese frühen Sorgen verharrten bei diesen vereinzelten Tierarten und Vorfällen und führten zu keiner Sensibilität für das Meer als Entität. Im Epilog steht dann der zeitverzögerte, gegenwärtige Bedeutungsgewinn des Ozeans als bedrohte Umwelt im Vordergrund. Diesem Wandel, der die menschliche Aufmerksamkeit auf die Gefährdung der Meere lenkte, ging eine Sensibilisierungsphase voraus. Die Taucher, Filmemacher und Schriftsteller, die den Ozean in den Jahren davor aufschlossen, erscheinen als bebildernde Wegbereiter des aquatischen Zweigs der Umweltbewegung (zum Film auch: 
Crylen 2015). Dieser Weg lässt sich an den sich verändernden Funktionen des Meeres nachzeichnen, vom „industrial workplace“ zur „wilderness in need of human protection“" (S. 206, zur ocean wilderness auch Kroll 2008).

In der längeren menschlichen Beziehungsgeschichte zur See zählt Rozwadowski drei größere Transformationen auf: Die erste Entdeckung der See („first discovery of the sea“) fand ab dem 15. Jahrhundert statt. Die Meere wurden zu einem workplace, einer militärischen Bühne und einem Transitraum geographischer Erkundung. Der zweite Zeitabschnitt („second discovery of the sea“) stand im Zeichen von Kunst, Literatur und zunächst küstenbezogener Freizeit- und Erholungsaktivitäten, die zur Wertschätzung führten (Gillis 2012: 128-157). Für die Gegenwart macht Rozwadowski eine "third discovery of the sea“ aus, „prompted by changes that have made the ocean and its depths more visible and culturally accessible." (S. 215). Diese dritte Entdeckung des Meeres basiert auf Sichtbarkeit und Partizipation. Spezielle Apps erlauben es den Menschen heute, den Wanderungen von Seeschildkröten und Haien zu folgen. Diese Formen von partizipativer Wissenschaft erhöhten das Miterleben und die Identifikation mit einem Teil des Planeten, der andernfalls jenseits der alltäglichen Reichweite läge.

Zusammenfassend lässt sich feststellen, dass alle vier Studien aufzeigen, wie wichtig eine Analyse des Meeres als Umwelt und Geschichtsraum ist. Die Konsolidierung des Meeres als Forschungsfeld macht deutlich, dass die jüngste historiographische Herausforderung der Einbezug der dritten Dimension des Planeten ist. Diese Perspektive hat gerade angesichts der dramatischen Veränderungen der Erde (Artensterben, Verschmutzung, Klimawandel) wissenschaftspolitische Berechtigung. Wenn diese Aufforderung ernst genommen wird, bedeutet es auch eine Neujustierung der Geschichten: Naturräume wie Meere erzählen in ihrer Entwicklung und ihrer materiellen Beschaffenheit andere als rein menschgemachte Geschichten. Gerade die gegenwärtigen Problemlagen erfordern diese kritische Auseinandersetzung mit der Umwelt der Meere und mit deren Aneignung durch die unterschiedlichen Akteure. Wie Jens Ruppenthal bemerkte, wäre hier die (in Vergessenheit geratene?) Sicht der longue durée nötig, die ihm zufolge im Meer bis heute „ein echtes Desiderat“ darstellt Ruppenthal (2018: 17).

\section{Anmerkungen}

1 Zur historischen Dimension der Einteilung der Meere und Ozeane: Lewis (1999).

2 Zur Klärung, welche Facette hier mit dem schillernden Begriff der Moderne gemeint ist, sei verwiesen auf James Scotts Verständnis von Moderne: Scott (1998). 
3 Die Bühne für spezialisierte Fragen der Umwelt-, Wissens- und Technikgeschichte stellte die ältere maritime history bereit. Sie stellt die zivile und militärische Schifffahrt in das Zentrum. Die historische Dimension der Schifffahrt (z. B. Entwicklung von Schiffen und Instrumenten) spielte seit Jahrhunderten eine Rolle, jedoch bedeutete der europäische Imperialismus einen Kulminationspunkt der Fokussierung auf die Seefahrt. Imperiale Flottenausstellungen und Meeresmuseen stellten den Anfang der systematischen maritime history in der Moderne dar. Die maritime history hatte seit jeher Berührungspunkte mit globalhistorischen Fragen. In den letzten zehn Jahren erfährt dieses Potential zunehmend historische Reflexion, siehe etwa Ruppenthal (2018: 30-37) und Polónia (2010: 1-20).

4 In der Bloomsbury-Reihe zur Kulturgeschichte erscheint im Jahr 2020 „The Cultural History of the Sea. From Antiqutity to the Global Age“.

5 Exemplarisch: Brunner (2011); Wessely (2013a, 2013b); Reiß \& Vennen (2014); Reiß (2020).

6 Zur Vorgeschichte der internationalen Umweltdiplomatie: Wöbse (2011). Am Beispiel von Walen, Dorsey (2013).

\section{Literatur}

\section{Verwendete Literatur}

Anand, Ram P. 1983. Origin and Development of the Law of the Sea. History of International Law Revisited. The Hague: Nijhoff.

Bauman, Zygmunt 2016. Moderne und Ambivalenz: Das Ende der Eindeutigkeit. Hamburg: Hamburger Edition.

Bolster, Jeffrey W. 2006. Opportunities in Marine Environmental. Environmental History (11): 567-597.

Bolster, Jeffrey W. 2012. The Mortal Sea: Fishing the Atlantic in the Age of Sail. Cambridge, MA: Belknap Press of Harvard University Press.

Brunner, Bernd 2011. Wie das Meer nach Hause kam. Die Erfindung des Aquariums. Berlin: Wagenbach.

Chiarappa, Michael und Matthew McKenzie 2013. New Directions in Marine Environmental History: An Introduction. Environmental History (18): 3-11.

Cowan, Robert C. 1960. Frontiers of the Sea. The Story of Oceanographic Exploration. New York: Doubleday.

Crylen, Jonathan C. 2015. The Cinematic Aquarium. A History of Undersea Film. Iowa City, Iowa: University of Iowa.

Cullather, Nick 2010. The Hungry World. America's Cold War Battle against Poverty in Asia. Cambridge, MA: Harvard University Press.

Dorsey, Kurkpatrick. 2013. Whales and Nations. Environmental Diplomacy on the High Seas. Seattle: University of Washington Press.

Dorsey, Kurk 2014. Crossing Boundaries: The Environment in International Relations. In: Andrew C. Isenberg (Hg.): The Oxford Handbook of Environmental History. Oxford: Oxford University Press: 688-715.

Finley, Carmel. 2011. All the Fish in the Sea. Maximum Sustainable Yield and the Failure of Fisheries Management. Chicago: University of Chicago Press.

Freestone, David (Hg.) 2006. The Law of the Sea. Progress and Prospects. Oxford: Oxford University Press.

Gillis, John R. 2011. Filling the Blue Hole in Environmental History. In: The Future of Environmental History: Needs and Opportunities: 16-18. RCC Perspectives 3.

Gillis, John R. 2012. The Human Shore: Seacoasts in History. Chicago: University of Chicago Press. 
Hardin, Garrett 1968. The Tragedy of the Commons. Washington, D.C.: American Association for the Advancement of Science.

Heidbrink, Ingo 2004. „Deutschlands einzige Kolonie ist das Meer!“. Die deutsche Hochseefischerei und die Fischereikonflikte des 20. Jahrhunderts. Hamburg: Convent.

Höhler, Sabine 2014. Exterritoriale Ressourcen. Die Diskussion um die Tiefsee, die Pole und das Weltall um 1970. In: Isabella Löhr und Andrea Rehling (Hg.): Global Commons im 20. Jahrhundert. Entwürfe für eine globale Welt. (Jahrbuch für Europäische Geschichte, 2014, Band 15): 53-82.

Holm, Poul 2012. World War II and the „Great Acceleration“ of North Atlantic Fisheries. Global Environment (10): 66-91

Hubbard, Jennifer M. 2006. A Science on the Scales. The Rise of Canadian Atlantic Fisheries Biology, 1898-1939. Toronto: University of Toronto Press.

Kraus, Alexander und Martina Winkler (Hg.) (2014a). Weltmeere. Wissen und Wahrnehmung im langen 19. Jahrhundert. Göttingen: Vandenhoeck \& Ruprecht.

Kraus, Alexander und Martina Winkler (2014b). Weltmeere: Für eine Pluralisierung der kulturellen Meeresforschung. In: Alexander Kraus und Martina Winkler (Hg.): Weltmeere. Wissen und Wahrnehmung im langen 19. Jahrhundert. Göttingen: Vandenhoeck \& Ruprecht: $9-24$

Kroll, Gary 2008. America's Ocean Wilderness. A Cultural History of Twentieth-Century Exploration. Lawrence: University Press of Kansas.

Lewis, Martin 1999. Dividing the Ocean Sea. Geographical Review (8): 188-214.

Lüttge, Felix 2016. Weniger schlechte Bilder. Walfängerwissen in Naturgeschichte, Ozeanographie und Literatur im 20. Jahrhundert. Berichte zur Wissenschaftsgeschichte (39): $127-142$.

Mackay, David 2018. In the Wake of Cook: Exploration, Science and Empire, 1780-1801. London: Routledge.

Nyhart, Lynn K. 2009. Modern Nature. The Rise of the Biological Perspective in Germany. Chicago: University of Chicago Press.

Polónia, Amélia 2010. Maritime History. A Gateway to Global History? In: Maria Fusaro und Amélia Polónia (Hg.): Maritime History as Global History (Research in Maritime History 43): 1-20.

Reiß, Christian 2020. Der Axolotl. Ein Labortier im Heimaquarium 1864-1914. Göttingen: Wallstein.

Reiß, Christian und Mareike Vennen 2014. Muddy Waters. Das Aquarium als Experimentalraum (proto-)ökologischen Wissen, 1850-1877. In: Kijan Espahangizi und Barbara Orland (Hg.): Stoffe in Bewegung. Beiträge zur Wissensgeschichte der materiellen Welt. Zürich: Diaphanes, 121-142.

Rozwadowski, Helen M. 2005. Fathoming the Ocean. The Discovery and Exploration of the Deep Sea. Cambridge: Belknap Press of Harvard University Press.

Rozwadowski, Helen M. 2012. Arthur C. Clarke and the Limitations of the Ocean as a Frontier. Environmental History (17): 1-25.

Ruppenthal, Jens 2018. Raubbau und Meerestechnik: Die Rede von der Unerschöpflichkeit der Meere. Stuttgart: Franz Steiner Verlag.

Ruppenthal, Jens, Ruth Schilling und Martin Weiss (Hg.) 2019. Von Flaschenpost bis Fischreklame. Die Wahrnehmung des Meeres im 19. und 20. Jahrhundert. Köln: Böhlau.

Sackel, Johanna 2017. Food justice, common heritage and the oceans. Ressource narratives in the context of the Third Conference on the Law of the Sea. International Journal of Maritime History (29): 645-659.

Scott, James C. 1998. Seeing Like a State. How Certain Schemes to Improve the Human Condition Have Failed. New Haven: Yale University Press.

Sparenberg, Ole 2012. „Segen des Meeres“. Hochseefischerei und Walfang im Rahmen der nationalsozialistischen Autarkiepolitik. Berlin: Duncker \& Humblot.

Steinberg, Philip E. 2001. The Social Construction of the Ocean. Cambridge: Cambridge University Press.

Turner, Jackson F. 1894. The Significance of the Frontier in American History. Madison: State Historical Society of Wisconsin.

Wessely, Christina (2013a). Der Ozean im Glas: Aquaristische Räume um 1900. Weinheim: Wiley. 
Wessely, Christina (2013b). Wässrige Milieus: Ökologische Perspektiven in Meeresbiologie und Aquarienkunde um 1900. Berichte zur Wissenschaftsgeschichte (36/2): 128-147.

Wigen, Kären 2007. Introduction. In: Jerry H. Bentley, Renate Bridenthal und Kären Wigen (Hg.): Seascapes. Maritime Histories, Littoral Cultures, and Transoceanic Exchanges. Honolulu: University of Hawai Press: 1-18.

Wöbse, Anna-Katharina 2011. Weltnaturschutz: Umweltdiplomatie in Völkerbund und Vereinten Nationen 1920-1950. Frankfurt/Main: Campus.

\section{Weiterführende Literatur}

Armitage, David and Alison Bashford 2014. Pacific Histories: Ocean, Land, People. New York: Palgrave Macmillan.

Bailyn, Bernard 2005. Atlantic History: Concept and Contours. Cambridge, MA: Harvard University Press.

Baucom, Ian 1999. Hydrographies. Geographical Review (89): 301-313.

Beer, Gillian. 2009. Darwins Plots: Evolutionary Narrative in Darwin, George Eliot, and Nineteenth-Century Fiction. Cambridge: Cambridge University Press.

Bentley, Jerry H. 1999. Sea and Ocean Basins as Frameworks of Historical Analysis. Geographical Review (89): 215-224.

Blum, Hester 2010. The Prospect of Oceanic Studies. PMLA (125): 670-677.

Blume, Dorlis, Christiana Brennecke, Ursula Breymayer und Thomas Eisentraut (Hg.) 2018. Europa und das Meer. München: Hirmer.

Böhnke, Günther und Arwed H. Meyl 1962. Denkschrift zur Lage der Meeresforschung: Im Auftrag der Deutschen Forschungsgemeinschaft und in Zusammenarbeit mit zahlreichen Fachgelehrten verfasst. Wiesbaden: Franz-Steiner-Verlag.

Bolster, Jeffrey W. 2008. Putting the Ocean in Atlantic History: Maritime Communities and Marine Ecology in the Northwest Atlantic, 1500-1800. The American Historical Review (113): 19-47.

Bose, Sugata 2009. A Hundred Horizons: The Indian Ocean in the Age of Global Empire. Cambridge, MA: Harvard University Press.

Braudel, Fernand 2001. Das Mittelmeer und die mediterrane Welt in der Epoche Philipps II. Frankfurt am Main: Suhrkamp.

Elvert, Jürgen 2018. Europa, das Meer und die Welt. Eine maritime Geschichte der Neuzeit. Müchen: Deutsche Verlags-Anstalt.

Lewis, Martin and Kären Wigen 1997. The Myth of Continents: A Critique of Metageography. Berkeley: University of California Press.

Rozwadowski, Helen M. 2002. The Sea Knows No Boundaries. A Century of Marine Science under ICES. Copenhagen: International Council for the Exploration of the Sea.

Hinweis des Verlags Der Verlag bleibt in Hinblick auf geografische Zuordnungen und Gebietsbezeichnungen in veröffentlichten Karten und Institutsadressen neutral.

\section{Franziska Torma}

Rachel Carson Center for Environment and Society

Leopoldstr. 11a

80802 München

Deutschland

franziska.torma@rcc.lmu.de 